\title{
Le transport de marchandises et de personnes sur le Nil en 823A.H./1420 È.C.*
}

\author{
Frédéric Bauden
}

La première ville que nous rencontrâmes s'appelle Fua [Fuwwa]. C'est une très grande ville et riche de toutes les choses que vous pouvez nommer. Elle se situe à l'opposé de l'île de Rosseto [Rashīd] [...]. Dans cette ville de Fua, nous nous arrêtâmes pour la nuit et mangeâmes à bord; dans la soirée, tous ceux de la ville, hommes et femmes, jeunes et vieux, vinrent pour [nous] voir, car ils sont peu habitués à voir des personnes comme nous [...]. Nous nous arrêtâmes dans cette ville pour la nuit et dormîmes à bord; puis nous quittâmes la ville en question à neuf heures le vendredi 7 octobre et nous continuâmes de remonter le Nil. ${ }^{1}$

Cette brève description d' un voyage sur le Nil donnée par Sigoli, pèlerin florentin qui souhaitait se rendre au Sinaï via Le Caire en 1384, fournit des indications précieuses sur les modalités qui régissaient un tel périple. Il faut bien avouer que de tels témoignages restent trop rares pour la période considérée et que nous sommes quelque peu démunis lorsqu' il s' agit d'étudier comment un voyage sur le Nil était organisé, dans quelles circonstances celui-ci se déroulait et à quel prix. Le document dont je propose la lecture et l'interprétation dans cette étude me permet précisément d'aborder la nébuleuse question du transport fluvial sur le Nil à l'époque mamlouke et d'apporter quelques réponses aux questions restées sans réponse jusqu’à ce jour.

* Je tiens à exprimer ma plus vive reconnaissance à Werner Diem pour sa lecture attentive et ses nombreuses remarques judicieuses. Il va de soi que je reste responsable des choix faits en dernier ressort.

1 Frescobaldi, Visit 165. J' ai traduit le texte italien.

(C) FRÉDÉRIC BAUDEN, 2015 | DOI:10.1163/9789004284340_008

This is an open access chapter distributed under the terms of the prevailing CC-BY-NC License at the time of publication. 


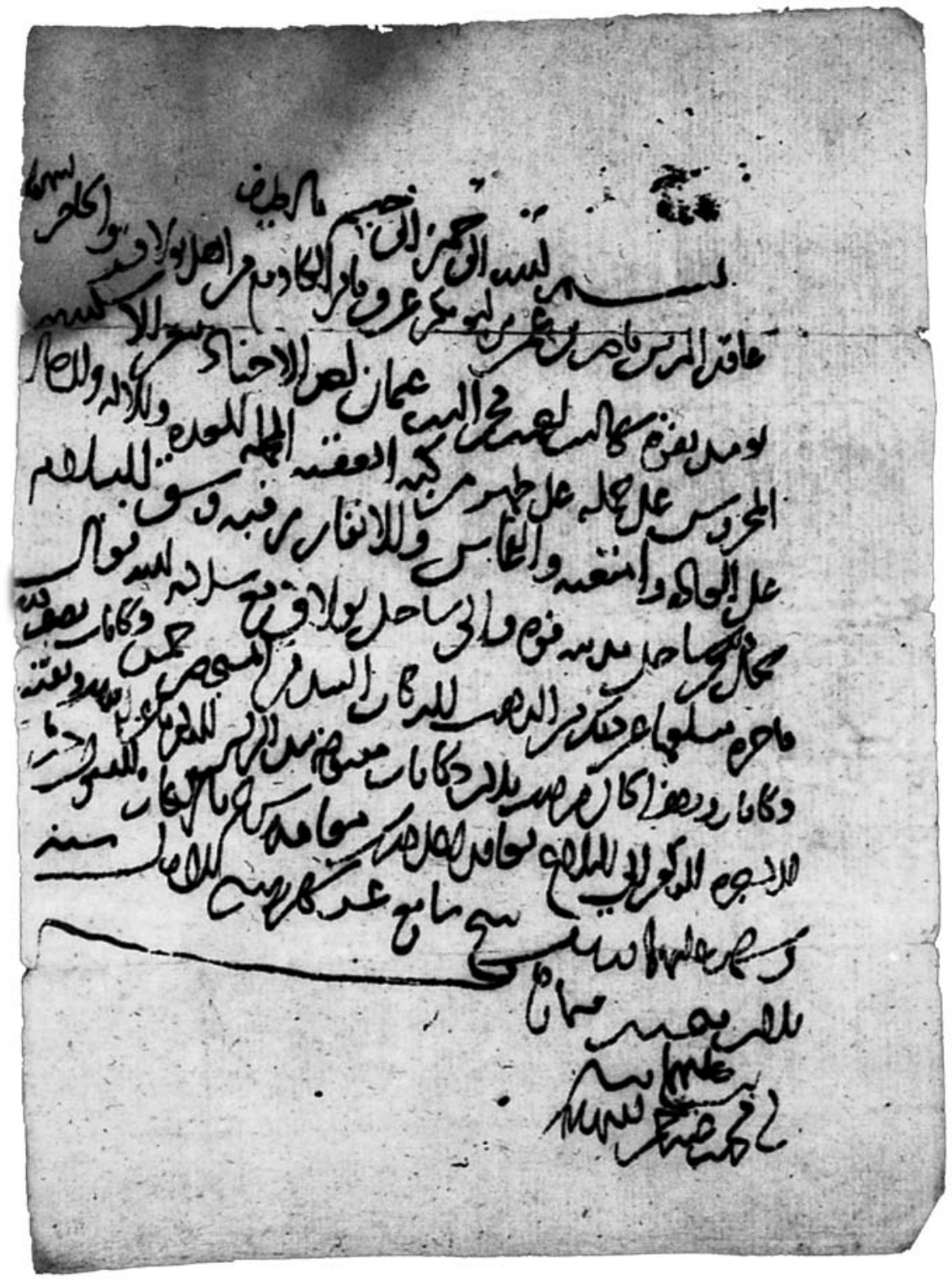

FIgURe 7.1 Procuratori di San Marco, Commissarie miste, busta 18o, fascicolo IX, no. 8. (C) Archivio di Stato di Venezia (ASVe) 


\section{Description $^{2}$}

Le texte est écrit, par une même main, à l' encre noire sur une feuille de papier mesurant 213 sur $155 \mathrm{~mm}$. La marge de droite occupe un espace qui oscille entre $29 \mathrm{~mm}$ (partie haute) et $24 \mathrm{~mm}$ (partie basse). La marge du haut, dans sa partie la plus large située au-dessus du $b \bar{a}$ ' de la basmala, mesure $25 \mathrm{~mm}$. Le papier est occidental avec fils de chaînette distants de $39 \mathrm{~mm}$ et parallèles au sens d'écriture. Les vergeures sont peu serrées. Le filigrane, coupé, ne permet plus son identification ${ }^{3}$. Le document porte quatre pliures horizontales séparées les unes des autres par un espace d'environ $5^{2-55} \mathrm{~mm}$. Ces pliures ne résultent

2 Une description sommaire du document avait été fournie dans l'inventaire des documents mamlouks conservés aux Archives de l'État à Venise. Voir Bauden, The Mamluk ${ }^{152-153}\left(\mathrm{n}^{\circ}\right.$ XI). Bien plus tôt, un résumé en avait été donné par Labib, Handelsgeschichte $502\left(n^{\circ} 9\right)$. Celui-ci comportait plusieurs erreurs qui sont corrigées dans la lecture qui suit.

3 Outre ce document, la même busta en compte deux autres écrits en arabe sur du papier occidental, parfois filigrané (les $n^{\text {os }} 2$ et 7 ). Voir Bauden, The Mamluk $15^{0-15^{1}}\left(\mathrm{n}^{\mathrm{o}} \mathrm{V}\right)$ et $15^{2}$ $\left(\mathrm{n}^{\mathrm{o}} \mathrm{x}\right)$.

J'ai déjà souligné l'importance de l'étude du matériau d'écriture pour les documents remontant à cette époque (Bauden, L'Achat d' esclaves 272). On connaît en effet encore fort peu dans quelle mesure le papier occidental était utilisé par la chancellerie d'état mamelouke ou pour la rédaction des actes privés, comme c' est le cas ici. Une étude d' ensemble des documents d'époque mamelouke permettrait sans doute de mieux appréhender la question, mais elle n' est pas sans soulever d'insurmontables problèmes techniques et administratifs, la majeure partie d'entre eux étant conservés dans des endroits fort peu accessibles aux chercheurs. Pour contourner ce problème, on peut avoir recours à l'étude des manuscrits datés ou datables à titre de comparaison. Une étude, qui a porté sur un ensemble cohérent (49o manuscrits du monastère de Saint-Macaire au Wādī l-Națrūn en Égypte), offre un éclairage qui n'est pas inintéressant (voir Zanetti, Filigranes). Au sein de cette collection, il apparaît que 80 manuscrits sont datés ou datables du XIII ${ }^{\mathrm{e}}$ au XV $\mathrm{XV}^{\mathrm{e}}$ siècle. (XIII $\mathrm{I}^{\mathrm{e}}$ siècle: 7 ; XIII ${ }^{\mathrm{e}}-\mathrm{XIV}^{\mathrm{e}}$ siècle: 4 ; XIV ${ }^{\mathrm{e}}$ siècle: 47 ; XIV $-\mathrm{XV}^{\mathrm{e}}$ siècle: 7 ; XV siècle: 15$)$, mais un seul, daté de 1420-1421, a été écrit sur du papier occidental (vénitien, en l'occurrence). À partir du XVI siècle, la balance s'inverse en faveur du papier occidental (essentiellement vénitien) avec deux manuscrits uniquement écrits sur du papier oriental (encore sont-il datés du début du XVI ${ }^{\mathrm{e}}$ siècle!). Voir Zanetti, Filigranes 446. On peut difficilement tirer des conclusions en l'absence d'analyses plus vastes. Pour les documents mamlouks conservés à Venise, j' avais déjà indiqué l' étrange absence de papier occidental avant le consulat de Biagio Dolfin (14181420), alors qu' il devient largement majoritaire pour les deux années en question. Ici encore, il faut se garder de conclusions hâtives, mais il est indéniable que l'importation de papier occidental est devenue plus importante qu' elle ne l' avait jamais été au tout début du XVe siècle. Voir Ashtor, Levant trade 210 et 597 (sub «paper exported to the Levant»). Intéressant à noter à plus d' un titre, le papier produit dans la péninsule ibérique n' apparaît pas dans les échanges 
pas de l'écrasement du document après avoir été roulé sur lui-même ${ }^{4}$ : il a d'abord été plié en deux, puis en quatre. On constate la présence d'une trace de mouillure dans le coin supérieur gauche qui doit être ancienne puisqu' on la retrouve sur plusieurs des documents arabes d'époque mamelouke conservés dans la busta $180^{5}$. Il est plus que probable que ces documents ont pris l'eau après leur arrivée à Venise où ils sont restés dans les mains de Lorenzo Dolfin avant d'entrer en possession des Procureurs de Saint-Marc ${ }^{6}$. Malgré cette tache, le texte est préservé dans sa totalité et la lecture n' en est pas entravée. Quelques points diacritiques sont indiqués, mais on ne trouve aucune signe orthoépique. Dans l'ensemble, il s'agit d'une écriture cursive aisément déchiffrable qui présente toutefois de nombreuses ligatures et abréviations qui sont détaillées dans le commentaire. Le verso est blanc.

\section{Analyse $^{7}$}

Le 17 du mois de rabī' I en l' an 823 ( $1^{\text {er }}$ avril 1420), le patron Nāṣir ibn 'Umar ibn Abū (sic) Bakr, connu sous le nom de Ibn al-Kādd [?] et originaire de Būlāq, a conclu un contrat avec un militaire en poste dans le port d'Alexandrie nommé Shihāb al-Dīn Aḥmad ibn Fakhr al-Dīn 'Uthmān. Celui-ci prévoit que ledit militaire sera transporté avec ses bagages et le tissu qui l' accompagne de la rive de Fuwwa à celle de Būlāq sur le bateau, du genre 'aqaba, appartenant audit patron, pour un montant de 5 ducats vénitiens. La moitié exigible de cette somme avant le départ est fixée à 3 ducats. Le solde sera versé à l'arrivée. Un seul témoin, le notaire, a attesté la validité de la transaction.

commerciaux entre la Catalogne et les territoires mamlouks pour la période qui va de ca. 1330 à ca. 1430. Voir Coulon, Barcelone 307-427.

4 Contrairement à d' autres documents conservés dans la même busta. Voir Bauden, L'Achat 272.

5 Voir Bauden, L' Achat 272 et 304.

6 Voir Pedani, The Mamluk 141.

7 Cf. Labib, Handelsgeschichte 502: «Ein Transportvertrag zwischen einem Soldaten der alexandrinischen Garnison und dem Kapitän eines Nilschiffes, in dem der letztere sich gegen ein Entgelt in Höhe von 5,5 Golddukaten verplifchtet, Güter, Stoffe und Menschen (Einzelheiten werden nicht genannt) von Fuwwa (am Nilzweig Rosetta) nach Būlāq, dem Hafen Kairos, auf seinem Schiff zu transportieren. Bei Vertragsabschluß wurden 2,5 Dukaten bezahlt, die übrigen 3 Dukaten sollte der Kapitän nach der Ankunft in Būlāq erhalten. Der Vertrag wurde laut Gesetz und in Anwesenheit eines Zeugen aufgesetzt und mit dessen Unterschrift versehen. Datum 17. Rabī‘ I 803/6. November 1400 ». 


\section{Texte}

$$
\begin{aligned}
& \text { بسم الله الرحمن الرحيم يا لطيف } \\
& \text { r) عاقد الريس ناصر بن عمر بن ابو بكر عرف بابن الكاد \}مع\{ من اهل بولاق والحاضر } \\
& \text { بشهو [0د] } \\
& \text { r) يوهـ/يـ/لذ بفوة شه(اب) الدين احمد بن فر الدين عثمان احد الاجناد بثغر الاسكندرية } \\
& \text { المحروس على حمله على ظهر مركه العقبة المحملة العدة والالة والرجال } \\
& \text { 0) على العادة وامتعته والقهاش وللانفار رقبة وست السلامة } \\
& \text { 7) يحمل ذلك من ساحل مدينة فوة والى ساحل بولاق مع سلا (مـ/مة الله تعالى } \\
& \text { V ) باجرة مبلغها عن ذلك من الذهب الدكات البندقي المشخص خمس دكاتات نصف } 8 \text { ذلك } \\
& \text { ^) دكاتان ونصف الحال من ذلك ثلاث دكاتات مقبوضة بيد الريس المذكور باعترافه بذلك وبقية }
\end{aligned}
$$

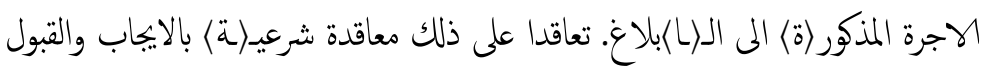

$$
\begin{aligned}
& \text { حسب ما [فيه|به] }
\end{aligned}
$$

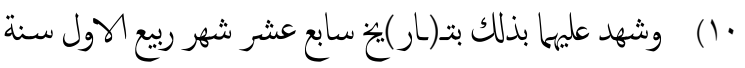

$$
\begin{aligned}
& \text { ثلاث وعشرين } 9 \text { وثمان ماية } \\
& \text { شـ(هد)ت عليها بذلك } \\
& \text { كتبه محمد بن عبد(مد الـ)رحمن البهائي }
\end{aligned}
$$

\section{Points diacritiques}

( ) الرحمن ؛ الرحيم ؛ لطيف Y) عاقد ؛ ابو ب) نفوة ؛ عمان ؛ الاحناد ع) مركه؛ العقه 0) وامتعهه ؛ والقاس ؛ ولانفار ؛ رفبه ؛وسق 7) فوه ؛ ولاف ؛ تعالى م) وبصف ؛ معوضه ؛ وققه 9) الاجره ؛ المي.

8 S. Labib s' est fourvoyé lorsqu'il parle d'un montant de 5,5 ducats d'or. Son erreur vient de ce qu'il a cru que la moitié payée était de 2,5 ducats et que le solde était de 3 ducats, alors que l'acompte est de 3 ducats et la moitié de la somme totale n'est indiquée que pour éviter toute falsification de cette somme. Le solde de deux ducats était payable à l' arrivée. Voir Labib, Handelsgeschichte $5^{02}$.

9823 et non 803 comme proposé par Labib, Handelsgeschichte 502. 


\section{Notes textuelles}

1. Il peut arriver qu' une formule laudative adressée à Dieu ou invoquant sa bénédiction sur le prophète soit ajoutée à la basmala, bien que cette pratique reste relativement rare. On peut citer à titre indicatif les exemples suivants: hasbunā llāh wa-ni'ma l-wakil (P.Cair.Arab. v 309, datable du III $/ \mathrm{IX}^{\mathrm{e}}$ siècle); al-ḥamd li-llāh wahdahu (Chrest.Khoury. I 78, datable des III ${ }^{\mathrm{e}}-\mathrm{IV}^{\mathrm{e}}$ siècles/IX ${ }^{\mathrm{e}}$ $\mathrm{x}^{\mathrm{e}}$ siècles); tawakkaltu alā llāh (P.Cair.Arab. I 37 = Chrest.Khoury. I 21, daté de 393/1003); al-ḥamd li-llāh wa-ṣallā llāh 'alā nabiyyinā Muhammad wa-'alāa ālihi al-țayyibìn wa-sallama taslìman (Chrest.Khoury. I 25, daté de 419/1028); wa-mā tawfíqū illà bi-llāh 'alayhi tawakkaltu wa-huwa rabb al-'arsh (Chrest.Khoury. II 1, daté de 444/1052); wa-mā tawfíqī illā bi-llāh 'alayhi tawakkaltu wa-ilayhi unību (P.Cair.Arab. I 68, daté de 459/1067); allāh al-muwaffiq li-l-ṣawāb (P.Cair.Arab. I 72, daté de 46o/1068); wa-l-hamd li-llāh ta'ālā (Bauden, L'Achat daté de 818/ 1415); wa-bihi nasta ìn (ASVe, Commissarie miste, busta 180, fascicolo IX, no. 2, daté de 821/1418); wa-l-hamd li-llāh wahdahu (Bauden, L'Achat daté de 822/ 1419); wa-ṣalātuhu 'alā sayyidinā Muhammad wa-ālihi (Bauden, The role daté de 822/1419); wa-huwa hasbī (CPR XXVI 45, daté de 874/1470); wa-l-hamd li-llāh (CPR XXVI 35, daté de 887/1482).

La basmala est ici suivie d'une invocation consistant en la particule du vocatif et un des quatre-vingt-dix-neuf noms de Dieu (lațif $)^{10}$. Cette formule, au moins attestée dans deux documents découverts au Haram de Jérusalem, reste rare $^{11}$.

2. Al-Rayyis. Comme le précise S. Hopkins ${ }^{12}$, les mots respectant le schème faćl se transforme, en moyen arabe, en fayül qui donne ensuite fayyil. Il ne relevait qu'un hapax (layyim), mais la forme que nous avons ici pour ra'̄s > rayyis est bien connue puisqu'elle figure même dans les dictionnaires classiques ${ }^{13}$. Le mot est répété avec le même ductus à la ligne 8 .

Ibn Abū Bakr. Pour le non-respect de la déclinaison du mot $a b \bar{u}$ en état d'annexion, voir Hopkins, Studies $§ 162$.i.

10 Elle n'a pas ici le sens de «Ô mon Dieu!», «Pour l'amour de Dieu!», qui est attesté par ailleurs. Voir Wehr, A dictionary 868 (s.v. لطف).

11 Jérusalem, al-Ḥaram al-Sharīf, docs. 1 et 308. Voir Little, A catalogue 31 et 34. On en trouvera un autre exemple dans un document rédigé par Ibn Ḥijja al-Ḥamawī (m. 1434), Qahwat al-insh $\bar{a}, 120$ (lațîf sans la particule du vocatif).

12 Hopkins, Studies $§ 24 a$.

13 Voir Lane, vol. III, 996. Cette forme était d' ailleurs utilisée en chancellerie. Voir al-Qalqashandī, Șubḥ al-a shāa, 6 :14. 
Ibn al-Kädd [ma'a]. Il s' agit d' une lecture conjecturale. La lettre située après l'alif ressemble plus à un $d \bar{a} l / d h \bar{a} l$ qu'à un $r \bar{a}$ tandis que la lettre finale présente un tracé souvent rencontré pour le 'ayn/ghayn dans cette position (voir toutefois la forme que prend précisément cette lettre dans la même position dans les mots $m a^{c} a(\mathrm{l} .6)$, sābic et $\left.r a b \iota^{c}(1.10)\right)$. On peut alors considérer que $m a^{\prime} a$ ne fait pas partie de la nisba et que la préposition fut écrite erronément par le notaire qui n' avait plus en tête qu' il avait introduit son texte par le verbe 'ãqada (transitif direct). Il n' aurait toutefois pas raturé le mot fautif au moment de la lecture. Une telle nisba n' est attestée ni dans les répertoires onomastiques ni dans les documents publiés jusqu'à présent. S. Labib l'avait interprétée comme étant al-Kārimī, lecture qui s' en rapproche, bien que moins convaincante, et qui fait référence à la catégorie des marchands d'épices, les Kārim ${ }^{14}$. La nisba est par ailleurs attestée ${ }^{15}$ et il n' aurait pas été impossible que le fils de l' un d' entre eux eût dû se reconvertir dans le transport fluvial pour des raisons économiques ${ }^{16}$. Il n'en reste pas moins que cette hypothèse perd tout son crédit quand on étudie attentivement le ductus.

$B i$-shuhū $[d i h i]$. Le mot est écrit en bout de ligne, légèrement surélevé, comme cela arrive fréquemment dans les documents de cette époque. Les deux dernières lettres ne sont pas visibles sur le document, mais elles pourraient être contenues dans le trait droit qui part de la base inférieure du $w \bar{a} w$. Le notaire a manifestement manqué de place et il est probable qu' il a dû ajouter ce mot $a$ posteriori.

3. Yawma('i)dh. Le ductus ne présente aucune marque visible de la lettre $y \bar{a}$ utilisée dans ce cas pour comme support de la hamza.

14 C' est ce que démontre une note de Fischel 1958, Über die Gruppe 168 (note 2 : « According to another communication from Prof. Cahen, Dr. Labīb has found in the Archives of Venice a document of the year 1400 pertaining to an agreement between a Kārimī and a shipcaptain for the transportation of commodities to Egypt with many interesting details»). Il s'agit bien du document décrit pas S. Labib dans son ouvrage sur le commerce en Égypte (Labib, Handelsgeschichte) dont j' ai cité le résumé plus haut (voir note 8). S. Labib avait lu la date comme étant $803 / 1400$, mais il avait passé sous silence cette interprétation de la nisba. D'autre part, c'est le patron de l'embarcation qui porte cette nisba, contrairement à ce qui est avancé par Fischel sur base d'une communication orale de $\mathrm{Cl}$. Cahen.

15 Voir Wiet, Les Marchands 107 et al-Ashqar, Tujjār 472 (Maḥmūd ibn al-Kuwayk ibn Karīmī [!]). Voir aussi Sublet, 'Abd al-Lațîf.

16 Sur les Kārimīs, voir ibid.; Fischel, Über die Gruppe; Labib, Handelsgeschichte; Ashtor, The Kārimī; Fischel, The spice; Goitein, New light; Labib, Les Marchands; al-Ashqar, Tujjār. 
Shihāb al-Dīn. Le ductus de ce laqab correspond à une abréviation où l'alif et le $b \bar{a}$ ont manifestement disparu, les deux mots étant liés ensemble.

Fakhr al-Dīn 'Uthmān. Le laqab aurait aussi pu être lu Muhyī l-dīn ${ }^{17}$, mais ce dernier n' est jamais combiné avec l' ism 'Uthmān alors que Fakhr al-Dīn l' est presque systématiquement ${ }^{18}$.

Ahad. L'alif est lié au ḥa .

6. Dhālik. Le mot fut clairement ajouté après lecture du document. Le notaire n'a cependant pas pris la peine de signaler cet ajout supralinéaire en fin de document comme l' exigent les règles en vigueur en ce domaine ${ }^{19}$.

9. Al-Madhkūr(a). La tā̉ marbūṭa a été oubliée par le notaire qui ne semble pas s' en être aperçu au moment de la lecture ou plutôt n' a-t-il pas considéré cette faute d' accord comme rédhibitoire.

Al-[I]blägh. L'alif initial manque indubitablement et doit être restauré, car le mot balägh ne signifie jamais «arrivée, destination.» On trouverait plutôt, pour cette acception, le terme bulügh. Iblägh laisse sous-entendre l'idée de livraison, de mener à destination qu' on ne peut traduire ici que par «arrivée » ou, dans un sens plus libre, « débarquement.»

Shar 'iy $(a)$. L'adjectif ne semble pas porter la marque de l'accord féminin avec le nom dont il dépend (mu'āqada). Le y $\bar{a}^{2}$ descend manifestement sous la ligne. S' il avait été suivi de la tā'marbūța, sa forme eût été différente.

Hasaba mā [bihi/fíhi]. Le mot apparaît à la suite de la formule légale d' offre et d'acceptation et juste avant celle de l'attestation. Il ne fait aucun doute qu' il est lié à celle qui le précède. De plus, le ductus n'offre guère de possibilités d'interprétation. Toutefois, la leçon que je propose reste conjecturale, car, à ma connaissance, elle n' est corroborée par aucun autre exemple attesté ${ }^{20}$.

\footnotetext{
17 Comme je l' avais pensé dans un premier temps (Bauden, The Mamluk 153).

18 Voir Malti-Douglas, The interrelationship 41.

19 Voir al-Nuwayrī, Nihāyat al-arab 9:8.

20 La leçon $j a z m^{a n}$, à laquelle j' avais pensé, est improbable. Elle n' aurait été corroborée par aucun autre exemple, à l' exception de son emploi dans le langage juridique: on parle de ḥukm jazm quand le jugement est irrévocable. Le terme aurait donc été employé comme adjectif, mais sa forme correspond ici au mașdar. Dans le cas qui nous occupe, ce dernier eût donc apparu dans la fonction d'un zarf. On trouve encore l'adjectif jāzim couplé à amr, pour désigner une chose décidée, ferme. Voir Amari, I Diplomi 209 (l. 5), 217 (l. 6), 229

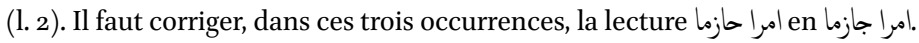


10. Bi-t $(\bar{a} r) \bar{c} k h$. Abbréviation que l' on trouve couramment dans les documents d'époque mamelouke ${ }^{21}$. La signature du notaire présente de nombreuses abréviations. La lecture de sa nisba est proposée par conjecture.

\section{Traduction}

1 Au nom de Dieu le Tout-miséricorde, le Miséricordieux. Ô [Toi qui es] Bon!

2 Le patron Nāșir ibn 'Umar ibn Abū Bakr, connu sous le nom d' Ibn al-Kādd $\{$ avec\}, habitant à Būlāq et en présence de ses témoins ce jour à Fuwwa, a conclu un contrat avec Shihāb al-Dīn Aḥmad ibn Fakhr al-Dīn 'Uthmān, qui est soldat dans la place protégée d'Alexandrie, pour le transporter sur le pont de son embarcation [du type] al-'aqaba, qui est chargée de l'équipement, des accessoires et de l'équipage

5 comme à l' accoutumée, ainsi que ses effets et le textile, les individus ayant des biens personnels/un capital, [et l'embarcation étant] chargée avec sûreté.

$6 \quad$ Il portera cela de la rive de la ville de Fuwwa jusqu'à la rive de Būlāq à la grâce de Dieu Très-Haut

7 pour un salaire dont le montant pour cela en ducats d' or vénitiens à figures sera de cinq ducats, la moitié de cela étant

8 deux ducats et demi, la moitié de la partie exigible de cela étant de trois ducats que le patron susdit reconnaît avoir reçus. Le solde

9 du salaire susdit sera [payé] à l' arrivée. Ils ont tous deux conclu un accord légal sur cela par offre et acceptation selon ce qu'il [le document] contient.

10 Témoignage a été pris de cela pour eux à la date du dix-sept rabīi I de l'an

11 huit cent vingt-trois [ $/ 1^{\mathrm{er}}$ avril 1420$]$.

J'ai témoigné de cela pour eux

Muḥammad ibn 'Abd al-Raḥmān al-Bahā'ī l' a écrit.

\section{Commentaire diplomatique}

Dans sa récente étude sur le droit maritime, $\mathrm{H}$. Khalilieh s' interroge, à juste titre, sur l'existence de contrats de transport maritime mis par écrit étant

21 Voir Bauden, L' Achat 273. 
donné qu'aucun document de ce type n'a été mis au jour. Sur base de cet argument ex silentio, il avance l' hypothèse que ces contrats maritimes devaient être majoritairement conclus oralement, en partie aussi à cause de l' illettrisme qui devait frapper les parties contractantes ${ }^{22}$. Ce dernier argument est battu en brèche par la correspondance, plus qu'abondante, se référant aux activités commerciales des marchands que les documents de la Genizah du Caire, et plus récemment de Qușayr al-Qadìm, ont permis de mettre en évidence ${ }^{23}$. La question de l'absence de contrats de transport trouve, quant à elle, une réponse dans le document qui fait l' objet de cet article. Unique en son genre, celui-ci démontre que de tels contrats écrits existaient bel et bien. Plus intéressant encore, il nous permet d'étudier le formulaire de ce type de contrat de location particulier. Pour le droit musulman, il appartient à la catégorie des documents relatifs à la location (al-ijāra), matière à laquelle les juristes ou les notaires consacrent un chapitre dans leurs textes juridiques ou leurs recueils de modèles. Pour la période concernée, c'est l'ouvrage d'un auteur presque contemporain, al-Asyūṭī (adhuc viv. 889/1484), qui fournit les données les plus pertinentes ${ }^{24}$. Étant égyptien, ce dernier était confronté quotidiennement aux problèmes que posait la rédaction de ce genre d'actes, fût-ce pour le transport fluvial ou maritime. Dans le chapitre consacré à la location, il aborde brièvement cette question en fournissant deux modèles de contrats ${ }^{25}$ : un premier consacré à la location d' une embarcation ${ }^{26}$ et un second, plus restrictif, ne touchant que le transport de marchandises sur une embarcation ${ }^{27}$. Ce sont ces modèles qui vont nous servir de fil rouge pour l'étude diploma-

22 Khalilieh, Admiralty 87 : «The level of literacy probably played a key role in the ratification of verbal contracts in courts. Since illiterates made up the great majority of the population, they created difficulties for the legal and administrative systems. As a matter of fact, the overwhelming majority of shipping contracts in the Mediterranean during this period were probably oral and dependent upon consent of the parties. This would explain why very little written evidence has survived. Written contracts were of course drawn up and used as forms of proof in case of legal altercations among the contracting parties. In short, whether the contract was to be oral or in writing was decided by the parties themselves ».

23 Pour les documents de la Genizah, on verra Goitein, A Mediterranean society. Pour ceux de Qușayr al-Qadīm, voir Guo, Commerce.

24 al-Asyūṭī, Jawāhir al-'uqūd.

25 Goitein, A Mediterranean society 1:233. Ces deux modèles ont été traduits par Khalilieh, Islamic 6o-61; Khalilieh, Admiralty 89-90.

26 al-Asyūṭ̂i, Jawāhir: șūrat ijārat markab. Cf. al-Samarqandī, Kitāb al-Shurūṭ 279.

27 al-Asyūṭī, Jawāhir: wa-in kāna l-ittifāq 'alā ḥaml shay' mu'ayyan min makān mu'ayyan ilā makān mu'ayyan daf'a wāhida. 
tique du document conservé à Venise. Ils nous permettront d'établir si celuici respecte le formulaire recommandé par al-Asyūṭī et, si oui, dans quelles mesures.

2. 'Áqada. Le terme introductif consiste en un verbe qui exprime la nature de la transaction. Il s' agit d'un contrat synallagmatique comportant des clauses réciproques, dont la plus importante est celle de l'offre et de l' acceptation ${ }^{28}$. Par conséquent, les deux parties s'accordent pour remplir leurs devoirs et obligations réciproques. La location (ijära) constitue une des catégories qui entrent dans le cadre du contrat ('aqd $)^{29}$. Si le contrat concerne la location d' une embarcation, donc d'un objet, celui-ci doit débuter par le terme istajara. Lorsqu'il ne porte que sur le transport de marchandises ou de personnes, en conséquence d'un service, c' est le terme 'ãqada qui est préconisé30.

Al-Rayyis. Conformément aux règles juridiques portant sur l'identification des parties, le document donne une description assez précise des deux personnes concernées. La première de ces parties correspond à la personne qui est propriétaire du bien, en l'occurrence le patron de l'embarcation désigné au moyen du terme rayyis. À côté des termes múallim, rubbān et nākhūdhā, plus fréquemment employés dans la littérature touchant à la navigation ou dans les documents, notamment de la Genizah, le mot rayyis s'est généralisé pour désigner le chef du navire et, par conséquent, celui qui est responsable de sa conduite, sans qu' entre en question le problème de la propriété ${ }^{31}$. Il est attesté, avec ce sens, déjà dans un document de la Genizah ${ }^{32}$. La description comporte l'obligation de donner le nom des personnes impliquées ainsi que leur filiation. Dans cette filiation, il est rare de trouver le nom du grand-père ${ }^{33}$, ce qui est pourtant le cas ici. À cela s' ajoute la shuhra, comme l'indique la formule 'urifa bi («connu sous le nom de»), qui est aussi recommandée quand elle existe ${ }^{34}$. Dans notre cas, il est probable que cette appellation courante joue ici un autre rôle. Dans le cas des patrons et des propriétaires de navires,

28 Voir plus bas, commentaire pour la l. 9.

29 Pour la théorie légale du contrat, voir Rayner, The theory 87-88, 100-101.

30 al-Asyūṭī, Jawāhir.

31 Voir Khalilieh, Islamic 42; Diem et Radenberg, A dictionary s.r. r's. Le mot rayyis s' est imposé au détriment des autres dans le dialecte égyptien. Voir Colin, Notes 75 (qui signale, à côté de la prononciation usuelle, une prononciation emphatique du $\sin$ ).

32 T.s. 12.434 (rayyis al-markab). Voir Khalilieh, Islamic 43 (note 26 ).

33 Rāğib, Actes de vente $16(\S 36)$.

34 Rāgib, Actes de vente 17 (§40). 
en effet, les documents de la Genizah prouvent que c'est souvent par leur nom courant que l' on faisait référence à leur embarcation ${ }^{35}$. Il n' est donc pas impossible que la shuhra qui figure dans notre document ait joué ce même rôle.

2-3. Min ahl Būlāq wa-l-hạạdir bi-shuhūdihiyawma’idh bi-Fuwwa. La description va plus loin encore en précisant le lieu d' origine du patron et en insistant sur sa présence au moment de la conclusion du contrat. Excès de précaution dans le chef du notaire? En tout cas, il a clairement ressenti le besoin de préciser ce point, bien qu' un contrat ne puisse être conclu en l' absence d'une des parties, à moins que l'une d' elles ne soit représentée par un mandataire ${ }^{36}$.

La deuxième partie contractante, le passager, subit le même traitement. On notera toutefois que seul le nom de son père est fourni, mais qu' au contraire du patron, son surnom (laqab) ainsi que celui de son père sont donnés.

3-4. Ahad al-ajnād bi-thaghr al-Iskandarīya al-mahrūs. Le passager possède une fonction: c'est un soldat (jundī) en fonction dans le port d'Alexandrie.

4. Alā ḩamlihi 'alā zahr markabihi. Après la mention de la nature de la transaction (contrat, múāqada) et les noms des parties, al-Asyūṭī poursuit son modèle par la formule 'alà an yahmil lahu 'alā zahr markabihi. Cette formule est presque identique à celle qui figure dans le document, à la différence que le notaire a préféré utiliser le mașdar et qu'ici il s' agit d'un passager et de ses bagages, et non du transport de marchandises uniquement, comme l' envisageait al-Asyūṭī.

Al-Aqaba. Dans la section sur la vente, al-Asyūṭī traite des embarcations dont il donne, d'ailleurs, une liste particulièrement détaillée pour la période considérée ${ }^{37}$. Comme tout bien destiné à la vente, une embarcation devait faire l'objet d'une description précise afin d'éviter toute plainte, quelle qu' elle soit, après la conclusion de la transaction. Cette précaution est également valable pour un contrat de transport, car le passager ou celui qui souhaitait faire transporter des marchandises devait être assuré que le voyage se ferait bien sur l'embarcation qu' il avait choisie. Le contrat était alors réputé conclu

35 Voir Goitein, A Mediterranean society 1:309 (exemple d'une embarcation nommée Ibn alIskandar d' après son patron, 'Alī ibn al-Iskandar), 312 (plusieurs exemples: Ibn al-Basmalī, Mufarrij, Ibn Naīm, Ibn Khallāf); Khalilieh, Islamic 27-29; Khalilieh, Admiralty 40-43.

36 Rayner, The theory $110-111$.

37 al-Asyūṭī, Jawāhir 1:78. 
pour une embarcation bien définie (safina bi-'ayniha $)^{38}$. Dans ce cas, le type d'embarcation devait être spécifié, sans entrer dans tous les détails requis pour la vente. Al-Asyūtịi, dans son formulaire de contrat de transport, utilise l' expression «markabihi al-fulānī,» renvoyant à la section sur la vente où il a précisément donné les différentes possibilités d'appellations pour les embarcations, qu' elles soient fluviales, maritimes ou habilitées à circuler à la fois sur le fleuve et en mer ${ }^{39}$. Dans le cas de ce document, l'embarcation correspond au type dit «al-'aqaba.» ${ }^{40} \mathrm{Ce}$ mot désignait un bateau qui ressemblait à une grande barque et que le voyageur Vincent Stochove décrivit de la sorte en 1631: «Les riches ont des basteaux exprés qui ne servent que pour ceste réjouissance publique [la crue du Nil], et les appellent Achaba, ils sont plats, la poupe en comprend plus de la moytié, elle est quarrée et entourée de balustres afin que ceux qui les mesnent n'incommodent les personnes qui sont assises dedans, elles sont par le bas couvertes de beaux et riches tapis de Perse, et le haut couvert de toile cirée, le dedans peint et diversifié par différentes sortes de couleurs, de façon que l'on y est comme dans une belle salle. $\gg^{41} \mathrm{~L}^{\prime}$ embarcation en question était courte et plate mais large, avec un voile carrée attachée à un mât central. La poupe était occupée par un château ou accastillage où se trouvait une grande salle garnie de tapis et de tissus. Il semble qu'à l'origine, elle était réservée au transport de la paille mais, avec le temps, elle fut aussi utilisée ponctuellement pour les festivités, entre autres celles liées à la crue du $\mathrm{Nil}^{42}$.

4-5. Al-Muhammala al-'udda wa-l-āla wa-l-rijāl 'alā l-'āda. C'est le mot al'aqaba qui précède, d' où l'accord féminin. Toutefois, on peut considérer que l' accord a été fait ici avec le mot markab qui, à cette époque, pouvait être des

\footnotetext{
38 Voir Khalilieh, Islamic 61-62; Khalilieh, Admiralty 89.

39 al-Asyūṭī, Jawāhir 1:233.

40 Ce type est mentionné par al-Asyūṭī, Jawāhir 1:78.

41 Stochove, Voyage en Égypte 34-35.

42 Voir Dozy, Supplément 2:146-147; Kindermann, «Schiff» 66-67; Colin, Notes 79; alNakhīlī, Al-Sufun 101-102. Voici la description précise qu' en donne le voyageur vénitien Alessandro Magno, qui l'emprunta entre Rosette et Būlāq en juin/juillet 1561: «On en trouve de deux sortes [germe]: l' une avec une voile latine se nomme cacabe, l' autre avec une voile carrée se nomme acabe. Presque toutes ont un mât de misaine. Au dernier niveau supérieur de la poupe, on érige des cloisons: il en est de même au niveau intermédiaire et les passagers construisent leurs propres abris sur cette poupe. Il en existe beaucoup de grande taille qui ont quatre voire six cabines sur cette poupe et parmi elles nombreuses sont celles qui jouent le rôle de navires. » Magno, Voyages 264 .
} 
deux genres ${ }^{43}$, même si c'est le genre féminin qui s' est finalement imposé, notamment dans le dialecte égyptien ${ }^{44}$.

Parmi les recommandations d'al-Asyūṭi pour la vente d'une embarcation, il indique qu'il y a lieu de préciser quels sont les équipements (' $\mathrm{udad}$ ) et les accessoires $(\bar{a} l a \bar{t} t)$ faisant partie intégrante de celle-ci et donc de la vente ${ }^{45}$, sans qu'il en dresse la liste. Celle-ci est par contre fournie par un autre formulaire rédigé par al-Jazīīī (m. 585/1189 $)^{46}$ : on y englobait le gréement, les voiles, le mât, les grapins, les cordes, les rames, etc ${ }^{47}$. Notre document se contente de préciser que l'ensemble de ces équipements et accessoires ainsi que l'équipage sont présents, sans en donner le détail, puisque le notaire s'en dispense en précisant que tous ces éléments sont conformes à l'usage ('alā l-'âa $a$ ).

5. Wa-amtiatihiwa-l-qumāsh. Outre le passager, on se doit également de signaler ses effets personnels et ses bagages. En cas de problème dû à une faute, il faut que le propriétaire des biens puisse obtenir réparation ${ }^{48}$. Le passager, dans le cas qui nous occupe, embarquait avec ses effets personnels (amtica) et du tissu ${ }^{49}$. Ces marchandises devaient normalement faire l'objet d'une description plus précise, particulièrement en matière de poids et de mesures, car le bon chargement de l'embarcation en dépendait ${ }^{50}$.

Wa-li-l-anfär raqaba. Cette expression n' est pas signalée dans les modèles de formulaires consultés. Elle se réfère évidemment à une possession des passagers désignés à l' aide du terme «individus.» Le mot raqaba couvre plusieurs acceptions. Il désigne, avant tout, le cou et, par synecdoque, une personne,

43 On trouve parfois des traces de cette ambivalence dans les textes où on passe du masculin au féminin dans une même phrase! Voir, par exemple, Archivio di Stato di Venezia, Procuratori di San Marco, Commissarie miste, busta 180, fascicolo IX, no. $5_{\text {(ll. 5-6: wașala }}$ al-thaghr markab lahā 'an al-Bunduqiyya muddat khamsinn yawm; 1. 9: hādhā l-markab). Les mss. dits «Galland» des Mille et une nuits, datables du xv siècle et originaires de Syrie, offrent d' autres exemples tout aussi parlants. Voir Halflants, Le Conte 116-117.

44 Colin, Notes 75 (note 3 ).

45 al-Asyūțī, Jawāhir 1:78. Cette recommandation est répétée succinctement dans la section sur la location (al-Asyūṭī, Jawāhir 1:233).

46 Dans al-Maqșid al-mahmūudfì talkhīṣal-'uqūd. On en trouvera la traduction dans Khalilieh, Admiralty 89.

47 Voir aussi Colin, Notes 62-75.

48 Voir Khalilieh, Admiralty 99-105.

49 Il ne semble pas que ce soit l' acception plus générale relevée par Amari qui soit d' application ici : marchandises en général ou petites choses. Voir Amari, IDiplomi 492 («mercanzie in generale e robe minute»).

5o Khalilieh, Admiralty 89 . 
puis un esclave ${ }^{51}$. Par métonymie, on en arrive à l' idée du droit de propriété $(\text { milk raqaba })^{52}$. On trouve aussi une autre acception plus technique tirée du sens initial: la peau du $\operatorname{cou}^{53}$ du chameau servait à fabriquer un sac destiné au transport de la poudre d' ${ }^{54}$. Dozy mentionne encore l' expression raqabat al-mäl dans le sens de capital ou somme d'argent ${ }^{55}$. De toutes ces significations, seules celles de la propriété en général ou du capital me paraissent les plus plausibles. On voit mal comment expliquer que tous les passagers étaient accompagnés d' un esclave. Par contre, le fait de préciser que chaque passager emportait avec lui des possessions, des biens personnels, ou encore que chacun d' entre eux possédait une somme d' argent, va de pair avec la formule qui suit. Entre ces deux possibilités, j' ai préféré ne pas trancher. En effet, les passagers étaient censés emporter tous les éléments qui devaient leur permettre de passer le voyage dans les meilleures conditions: vêtements, nourriture, toilette et couchage ${ }^{56}$ mais les passagers pouvaient aussi être soucieux de voir mentionner qu'ils voyageaient avec un capital. Dans les deux cas, ils devaient être en mesure d'obtenir réparation en cas de naufrage.

Il reste une autre possibilité de lecture: wa-l-anfār. Dans ce cas, il était accompagné de personnes, qui étaient peut-être celles qui gardèrent le contrat à l' arrivée: le consul vénitien et ses hommes qui l' accompagnaient. L' interprétation du mot qui suit reste alors tout aussi problématique.

Wasq al-salāma. Cette formule apparaît dans le premier modèle relatif à la location d'une embarcation que donne al-Asyūṭi dans son traité57. Elle vient préciser les mots qui précèdent dans ce formulaire: le locataire pourra jouir du bien loué en le chargeant avec sûreté. Cette clause est fondamentale: ici, elle garantit que le patron de la barque s'engage à ne pas charger son embarcation plus qu'il n' est permis. Pour éviter les surcharges, on marquait une ligne de flottaison sur chaque bateau destiné au transport de marchandises et de personnes. Tant que cette ligne restait visible au-dessus de l'eau, le chargement était considéré comme sûr ${ }^{58}$.

\footnotetext{
51 Lane, Madd al-Qāmūs 3:299 (s.v. رقبة).

$5^{2}$ Khalilieh, Admiralty 47.

53 Lane, Madd al-Qāmūs 3:300 (s.v. رقبّب et رقبة).

54 Dozy, Supplément 1:546 (s.v. رقبة).

55 Ibid.

$5^{6} \quad$ Khalilieh, Islamic ${ }^{6-57}$; Khalilieh, Admiralty 75.

57 al-Asyūțī, Jawāhir 1:233.

$5^{8} \quad$ Khalilieh, Islamic $3^{1-32}$; Khalilieh, Admiralty $3^{6-37 .}$
} 
6. Min sāhil .. wa-ilā sāhill. Après la mention des biens transportés, le formulaire prévoit que le contrat doit indiquer précisément les points de départ et d'arrivée. Celui-ci étant conclu pour un parcours déterminé, le montant réclamé couvre ce transport jusqu'à destination, quelles que soient les circonstances ${ }^{59}$. Al-Asyūṭī l' exprime en ces termes: min al-balad al-fulānī ilā l-balad al-fulānī. Notre document met en évidence que le transport se fait d'un port à un autre, exprimé au moyen du terme sāhil (rive), plutôt que d'une ville à une autre (balad). Dans le cas du point de départ, c'est de la rive de la ville (madìna) de Fuwwa qu'il s' agit, alors que pour l' arrivée, on ne parle que de la rive de Būlāq. Cela s' explique par le fait que Būlāq n'est pas une ville à part entière, mais un simple quartier du Caire.

Ma'a salāmat allāh ta'ālā. Ce transport est assuré à la grâce de Dieu. AlAsyūṭī préconise l' emploi de cette expression sous une forme plus complète: ma'a salāmat allāh ta'âlā wa-'awnihi (à la grâce de Dieu Très-Haut et avec Son aide). En d' autres termes, le transport fluvial ou maritime n' est assuré que dans la mesure où c' est Dieu qui est souverain. Le transport sur le Nil, à cette époque, est loin d'être une croisière: les éléments naturels peuvent toujours être la cause de retards ou de dégâts, parfois d'un naufrage. À ceux-ci s'ajoutent les imprévus comme les attaques de pirates qui exerçaient cette activité y compris sur le fleuve ${ }^{60}$.

7. Bi-ujra mablaghuhā. Les clauses du contrat déterminées, le notaire passe ensuite à la détermination du salaire ou de la rétribution qui est prévu pour le service rendu ou la location. La formule est conforme aux indications d' alAsyūṭī et se rencontre, par ailleurs, fréquemment dans les contrats.

Al-Dhahab al-dukāt al-bunduqī al-mushkhas. Conformément aux règles juridiques, le montant est dû dans une devise ou un numéraire qui doivent être spécifiés. Les documents conservés à Venise mettent en évidence, pour l'époque concernée, la prépondérance du numéraire vénitien, en l'occurrence le ducat ${ }^{61}$ : ASVe, Commissarie miste, busta 18o, fasc. IX, no. 2: dukāt dhahab firanjī; ASVe, Commissarie miste, busta 180, fasc. IX, no. 7: al-dhahab al-dukāt al-firanjī al-bunduqī al-wāzin; ASVe, Commissarie miste, busta 180, fasc. IX, no. 9: al-dhahab al-dukāt al-firanjī al-bunduqi ${ }^{-62}$. Que le numéraire, quand il

\footnotetext{
59 Khalilieh, Admiralty 132-133.

6o Khalilieh, Islamic 142-143.

61 Les propos d'un contemporain, E. Piloti, font écho à cette situation: «Et ne prent-on ducas se non qu'ilz soyent venitians». Voir Piloti, Traité 108.

62 Sur les cinq contrats conservés, seul un prévoit un paiement dans une devise locale: ASVe, Commissarie miste, busta 180, fasc. IX, no. 12: dirham fulüs.
} 
est étranger, soit uniquement vénitien et non florentin, n'a rien de surprenant puisque les transactions concernaient des Vénitiens essentiellement. D' autre part, le ducat vénitien jouissait à cette époque d'une renommée légèrement surfaite dans tout le bassin méditerranéen, et particulièrement en Égypte, au grand dam des sultans mamelouks. Deux ans avant la date de ce document de transport, al-Mu' ayyad Shaykh décidait de faire interdire l'usage du ducat pour les transactions ayant lieu sur ses territoires ${ }^{63}$. Cette prohibition ne fut pas vraiment suivie d' effets puisque les documents conservés à Venise font état de paiements effectués en ducats vénitiens dans les mois qui suivirent ${ }^{64}$.

Dans notre document, c' est toujours le ducat qui sert de monnaie de paiement, mais les termes utilisés pour le décrire diffèrent quelque peu de ceux rencontrés dans les trois autres témoins conservés aux Archives de l'État à Venise. Dans ces derniers, le ducat est toujours qualifié de vénitien (bunduqī) tout en étant décrit, préalablement, comme franc ( firanjī). L'appellation «vénitien» vient donc préciser le terme de «franc». Cette origine plus générale n' est pas soulignée ici. Par contre, la provenance vénitienne est renforcée par le mot qui lui est adjoint (al-mushkhaș) ${ }^{65}$. Le ductus ne laisse aucun doute quant à sa lecture. Ce terme fait référence aux figures qui ornaient le ducat vénitien sur ses deux côtés: à l' avers, le doge en position assise recevant le gonfalon des mains de saint Marc; au revers, le Christ. Al-Qalqashandī, dans la description qu'il en donne ${ }^{66}$, confond les deux personnages avec les saints Pierre et Paul. Le terme arabe recouvrait donc une connotation négative et, lorsqu' il fut décidé, en 829/1425, de mettre sur le marché une nouvelle monnaie d'or mamlouke, l'ashrafi, pour contrer la popularité du ducat et des autres monnaies d' or étrangères, c'est précisément ce terme qu'utilise Ibn Taghrī Birdī pour parler du monnayage des Francs «qui porte les insignes de leur infidélité.» 67

Nisf dhālik. Afin d'éviter la falsification du prix, la mention de la moitié du prix est requise, mais pas toujours respectée ${ }^{68}$. Elle apparaît bien ici.

63 Bacharach, The dinar 85-86.

64 Bauden, L' Achat 285.

65 Cf. Dozy, Supplément 1:735 renseigne aussi une autre vocalisation d'après le Tāj al-'arūs d' al-Zabīdī (mashkhaș). Popper (p. 46) donne mushakhkhaș sur la base d' Ibn Taghrī Birdī. Au XIX ${ }^{\mathrm{e}}$ siècle, ce terme (mushakhkhaș) est toujours en usage en Égypte où, en parlant de monnaie, il signifie « comptant». Voir Von Kremer, Beiträge 1: 82 (d' après al-Jabartī).

66 al-Qalqashandī, Șubḥ al-a'shā 3:441.

67 Popper, Egypt and Syria 1:46-47.

68 Voir Bauden, L' Achat 286; Rāgib, Actes de vente 43-44 (§113). 
8. Al-Hāll min dhālik. Le premier terme désigne la partie exigible au moment de la conclusion du contrat. Cette partie peut représenter le montant total ou partiel du prix convenu. Dans le cas qui nous occupe, trois ducats ont été réceptionnés par le patron qui reconnaît en avoir pris possession (maqbüḍa bi-yad ... bi-tiräfihi bi-dhālik).

8-9. Wa-baqīyat al-ujra al-madhkūr[a] ilā l-[i]blägh. Le montant payé ne représentant qu' une partie du prix total convenu, le solde sera versé à destination. Dans sa majorité, la littérature juridique prévoyait, pour ce genre de contrat, que le paiement total devait se faire à destination, du moins pour le transport maritime. Ce document établit que ce n'était pas toujours le cas. Bien au contraire, un paiement partiel correspondant au moins à la moitié du montant total était généralement réclamé, comme le prouvent les documents de la Genizah $^{69}$. Al-Asyūțī se démarquait en cette matière puisqu'il précise que le montant peut être perçu avant le départ soit en entier, soit partiellement, soit encore être payé en plusieurs fois ${ }^{70}$. La pratique contemporaine du document confirme donc le formulaire de ce dernier.

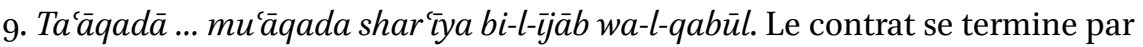
cette formule consacrée. En effet, l' offre et l' acceptation scellent la transaction, chacune des parties le faisant en connaissance de cause ${ }^{71}$.

11. Pour éviter tout ajout postérieur à la rédaction de l'acte, on constate que les notaires prenaient soin de terminer la ligne soit par une formule religieuse réservée à cet usage dans les documents (hasbunā llāhwa-ni'ma l-wakīl), soit de faire usage de la kashìda qui consiste à allonger le trait reliant les deux dernières lettres du mot terminant la phrase ${ }^{72}$. C'est ce dernier procédé qui est visible ici.

Dernier élément qui a son importance, deux témoins de sexe masculin ${ }^{73}$ au minimum, dont l'un était généralement le scribe et l'autre un témoin profes-

69 En cela, la pratique correspondait à celle en vigueur dans le droit maritime byzantin. Voir Khalilieh, Islamic 64-65; Khalilieh, Admiralty 125. Goitein avance plutôt l'idée que la majeure partie du paiement du frêt se faisait à l' arrivée, sauf pour le transport maritime où une avance était réclamée. Goitein, A Mediterranean society 1:312 et 342.

70 al-Asyūṭī, Jawāhir 1:233.

71 Bauden, L'Achat 286.

72 Ces deux systèmes sont indépendamment mis en œuvre dans les deux documents publiés dans Bauden, L' Achat.

73 C' est un verset coranique qui en constitue la base juridique indiscutable (II: 282). 
sionnel, du moins à l'époque qui nous concerne ${ }^{74}$, devaient attester de leur présence au moment de la conclusion du contrat. Comme on peut le constater, ce document a été rédigé par un unique témoin dont on trouve la signature au bas à droite. En cela, il était dépourvu de valeur probatoire en cas de litige, mais ne pouvait nullement être considéré comme caduc au regard du droit ${ }^{75}$.

\section{Commentaire historique}

Ce contrat de transport, conclu dans la ville de Fuwwa par un militaire en garnison dans le port d'Alexandrie en 1420, prévoyait qu' il serait emmené à la grâce de Dieu de cet endroit jusqu'à Būlāq, le principal point d' abordage à cette époque dans la capitale de l' empire mamlouk. Document unique en son genre, il permet de traiter la question du transport fluvial au début du Xve siècle. Pour ce faire, l' historien dispose de plusieurs sources que l' on peut classer comme suit:

- des documents conservés évoquent le transport sur le Nil de manière fragmentaire: il s' agit de notes de compte, de lettres qui donnent des renseignements assez précis sur l'itinéraire, la durée et les conditions du voyage, mais dont les données restent malheureusement parcellaires quant au coût du transport des personnes et des marchandises, car elles sont généralement globales ${ }^{76}$. De tels documents concernent essentiellement les périodes plus anciennes et figurent dans les riches fonds de la Genizah. Ils ont été particulièrement bien étudiés depuis les travaux de Goitein ${ }^{77}$. Les papyrus peuvent aussi apporter leur lot d'informations, bien que plus rarement ${ }^{78}$.

74 Il est incontestable qu'à l'époque mamelouke cette professionnalisation du témoin était bien établie. Voir Bauden, L' Achat 318.

75 Rāgib, Actes de vente 105 (§282).

76 Goitein, A Mediterranean society 1:339 («The details required for an exhaustive study of the subject - the ports of embarkation and destination, description of the consignment, its weight and value, the freight and the customs paid - are rarely given in full, and very often all the expenses incurred during a journey (namely, the freight, customs and other dues) are lumped together»).

77 Goitein, A Mediterranean society vol. I, chapitre IV (Travel and Seafaring); Udovitch, Time, the sea.

78 Sijpesteijn, Travel and trade (lettre datable de 117/735 donnant un itinéraire et la liste des frais encourus). 
- les ouvrages à caractère juridique (théorie légale, pratique du droit, recueils de modèles, collections de fatwa $\bar{a}$-s) fournissent le cadre dans lequel les transactions liées au transport fluvial sont conclues. S'ils sont avares en données factuelles (tous frais liés au transport), ils permettent de mieux comprendre comment le contrat était établi et quels étaient les devoirs et obligations des deux parties ${ }^{79}$.

- les ouvrages à caractère historique et géographique sont aussi particulièrement utiles pour les informations qu' ils donnent, fût-ce de manière anecdotique, sur les ports, les canaux et leur état, les itinéraires et les taxes ${ }^{80}$.

- enfin, les récits de voyage, rédigés soit par des voyageurs occidentaux, marchands ou pèlerins, ou par des musulmans généralement de passage en Égypte au cours du périple qui les conduisait au pèlerinage à La Mecque, restent des sources précieuses. Ces voyageurs étaient en effet souvent attentifs aux frais liés à leur séjour et ils ne manquaient pas une occasion pour se plaindre des exactions dont ils avaient été victimes ${ }^{81}$.

Ces sources mettent en évidence que le trajet Alexandrie-Le Caire (port de Fusțāt, puis, plus tard, Būlāq) pouvait être accompli de différentes manières et selon plusieurs itinéraires, selon les époques considérées:

- soit par voie fluviale d'un point à l' autre, en employant, entre Alexandrie et le Nil (bras de Rosette dit branche bolbitine), un canal;

- soit un trajet mixte, par voies fluviale et terrestre, à dos d'âne, de mule ou de chameau $^{82}$, ou par voies maritime (Méditerranée) et fluviale ${ }^{83}$;

- soit, enfin, uniquement par voie terrestre, la plus longue, la moins sûre et la plus coûteuse de toutes les possibilités.

79 Ces sources ont été particulièrement exploitées par Khalilieh, Islamic; Khalilieh, Admiralty.

8o Ces données ont été rassemblées assez exhaustivement par Toussoun, Mémoire sur les anciennes et Toussoun, Mémoire sur l'histoire. On complétera par al-Makhzūmī, al-Minhāj.

81 On verra le panorama très complet qui est donné de ces sources dans Hairy et Sennoune, Géographie historique et A. Graboïs, La Description.

82 C'était le cas de l' auteur de la lettre datable de 117/735: il voyagea de Fusțāṭ à Rosette par bateau et, ensuite, de Rosette à Alexandrie sur un âne ou une mule. Voir Sijpesteijn, Travel and trade 116. Sur la route entre Rosette et Alexandrie, voir Combe 1929.

83 Voir, par exemple, pour cette deuxième possibilité, le témoignage de Bernard de Breydenbach en 1484 apud Combe, Alexandrie 121. 
L'élément décisif pour déterminer quel itinéraire serait suivi et quels moyens de transport seraient employés était la saison. À l'époque considérée, la branche canopique qui reliait anciennement Alexandrie au fleuve était insuffisamment irriguée et trop ensablée pour permettre sa navigation sur tout son tracé. Outre le souci de permettre le transport des personnes et des marchandises de et vers Alexandrie par voie fluviale, considérée comme la plus rapide, il s' agissait aussi d'alimenter la ville d'Alexandrie en eau douce ${ }^{84}$. La solution apportée à diverses époques de l'ère musulmane consistait à augmenter le débit d' eau en procédant à des captages situés plus en aval ${ }^{85}$. Ces points de captage partaient tous de la branche bolbitine qui se jette à la mer à hauteur de Rosette ${ }^{86}$. Sous le règne du sultan al-Nāșir Muhammad ibn Qalāwūn, en 1310 précisément, le besoin se fit sentir de procéder à de nouveaux travaux pour combattre l' ensablement de la branche canopique. Un canal fut construit entre la branche bolbitine, à hauteur d' al-'Ațf, bourgade située sur la rive gauche du Nil vis-à-vis de la ville de Fuwwa, et l' extrême fin du parcours de la branche canopique, plus en aval donc, longeant ainsi le lac d' Edkü ${ }^{87}$. Ce canal, qui prit le nom de ce sultan (al-Nāṣirīya), joua son rôle pendant quelques décennies ${ }^{88}$ mais, en 1368 , il fallut déjà procéder à un curage ${ }^{89}$, signe que l' ensablement et la subsidence étaient inexorables. Si navigation il y avait, elle était indubitablement assurée pendant les mois de crue, entre avril et octobre ${ }^{90}$. En 1384, Frescobaldi et ses compagnons florentins quittèrent la ville d'Alexandrie le 5

84 Les nombreuses citernes de la ville étaient remplies durant la crue, à partir du printemps, et c' est sur ces réserves en eau que la ville vivait jusqu'à celle de l'année suivante. Voir Piloti, Traité 65-66 (même information fournie par Ghillebert de Lannoy en 1422, Piloti, Traité $65^{-66}$ note a).

85 Pour la géographie historique du Delta à l'époque musulmane, sur base des sources anciennes, voir Guest, The delta. On trouvera un tracé des différents canaux qui ont relié la ville d'Alexandrie au Nil à travers l'histoire dans Kahle, Zur Geschichte entre les pages 82-83; Hairy et Sennoune, Géographie 276.

86 Voir Hairy et Sennoune, Géographie 250.

87 Les travaux ont sans doute remis en fonction un ancien bras puisqu'il existait déjà une jonction entre Fuwwa et Alexandrie au XI ${ }^{\mathrm{e}}$ siècle. C' est ainsi qu' elle est mentionnée dans des documents de la Genizah. Goitein, A Mediterranean society 1:295, 297, 299.

88 Sigoli signale la présence d' une écluse sur ledit canal en 1384, signe que l' on pouvait régler le débit. Voir Frescobaldi, Visit 164.

89 Hairy et Sennoune, Géographie 250.

9o Il semble que ce fût le cas depuis au moins l' époque fatimide. Vers la fin du mois d' octobre 1140, un marchand informait son correspondant au Caire que d'ici peu le canal ne serait plus praticable pendant plusieurs mois. Voir Goitein, A Mediterranean society 1:298. 
octobre $^{91}$. Ils firent l'équivalent d' un mille à dos de mule et arrivèrent au canal où ils embarquèrent dans une germe (en ar. jarm, barque) qui les conduisit jusqu'à Fuwwa d'une traite ${ }^{92}$. Ces propos sont confimés par un autre voyageur, Brancacci, qui voyagea deux ans après notre soldat (1422), en empruntant le même itinéraire que Frescobaldi, toujours au moment de la crue: parti d'Alexandrie à cheval, le 30 août, il rejoignit un port situé, dit-il, à 3 milles de la ville où il embarqua sur une germe qui le conduisit jusqu'à Fuwwa ${ }^{93}$. L' année suivante (826/1423), le sultan Barsbāy décida d' entreprendre des travaux destinés à rendre ce canal navigable à nouveau toute l'année. L'entreprise dura 90 jours, mais son effet fut de courte durée $e^{94}$.

Sur base de ces données, il est donc établi que le canal partait à peu de distance d'Alexandrie et que le voyage se faisait en une traite jusqu' au point de captage situé à al-'Ațf, sans changer d' embarcation. En chemin, les voyageurs s'étonnent des nombreuses petites villes plus commerçantes qu'agricoles situées en bordure, conséquence de l'incessant va-et-vient de barques transportant tant marchandises que passagers ${ }^{95}$. À ce stade, l'embarcation marquait un arrêt le long de la rive de la ville de Fuwwa, qui est la plus souvent mentionnée dans les récits de voyages ${ }^{96}$. Elle est décrite comme une ville d'importance ${ }^{97}$ avec ses marchés et boutiques, entourée qu' elle est de nom-

91 En 1440, Piloti de Crète fait remarquer que les bateaux arrivent du Nil jusqu'à Alexandrie par ce même canal. Il s' agit à nouveau de la période de crue (septembre). Voir Piloti, Traité 183 («Au mois de septembre attendons en Dieu que aurons la terre d' Alexandrie, qui est au temps que le flume est creu, et sez sarme [= jarm], par la voye du Calis [= khalï], viennent jusques aulx murs de la terre»).

92 Frescobaldi, Visit 42. C'était encore le cas un an plus tard. Voir Kahle, Zur Geschichte 77-78. On signalera que des bateaux musulmans pouvaient évidemment remonter le Nil depuis la Méditerranée par l' embouchure de Rosette, et ce jusqu' au Caire. Voir Goitein, $A$ Mediterranean society 1:296. Ce fut aussi le cas pour des bateaux provenant du dāral-harb, sans doute sous bonne escorte, mais cette facilité leur fut déniée à partir des croisades. On en trouve encore des occurrences dans les documents de la Genizah (Udovitch, Time, the sea 522 ).

93 Brancacci, Diario 169. S' il faut en croire Gucci, les chevaux étaient normalement réservés aux militaires et aux gens d'un certain rang. Les voyageurs étrangers n' avaient le droit de chevaucher que des ânes ou des mules. Voir Frescobaldi, Visit 96.

94 Kahle, Zur Geschichte $78-79$.

95 Wiet, Les communications 244.

96 Graboïs, La description 533 .

97 Belon du Mans, en 1553, exagère sans doute quand il la dit presqu' aussi grande que la capitale. Apud Maspero et Wiet, Matériaux 141. 
breux vergers et de cultures en tout genre ${ }^{98}$, notamment de canne à sucre ${ }^{99}$. En 1384, Frescobaldi la définit comme une forteresse sans murailles ${ }^{100}$. D' après ses indications, on comprend que les bateaux qui arrivaient à cet endroit marquaient un arrêt pour remplir des obligations liées sans doute au fisc et à la douane. Les passagers passèrent la nuit à bord, y prenant aussi leur repas, pour éviter des frais supplémentaires ainsi que pour des raisons de sécuritée101. Il n'y eut pas de transbordement à Fuwwa et on comprend donc que c'est la même embarcation qui les conduisit d' Alexandrie jusqu' au Caire.

Le soldat qui conclut le contrat de transport fluvial était en garnison à Alexandrie. Si l'on considère qu'il a conclu ce contrat à Fuwwa, on peut légitimement se demander s'il a accompli le voyage séparant Alexandrie de la branche bolbitine en bateau. La date du contrat (1 avril 1420) indique que nous étions au tout début de la crue, à un moment où le débit du Nil n'était pas encore suffisant pour alimenter le canal jusqu'à Alexandrie. Il a donc dû prendre un autre moyen de transport, probablement le cheval, jusqu'à l'endroit où la navigation était possible. Le fait qu' il a dû conclure un contrat de Fuwwa au Caire démontre qu' il est soit arrivé directement à cheval à Fuwwa ou, s'il a pris une embarcation, qu'il devait en changer à cette jonction, car un nouveau contrat impliquait des frais supplémentaires et ne faisait qu' augmenter le coût du transport jusqu'à la destination finale. Dans ce cas, cela signifierait aussi que certaines embarcations étaient spécialisées dans la liaison reliant Alexandrie à Fuwwa, ce qui n' est pas impossible. Les témoignages des pèlerins occidentaux

98 Labib, Handelsgeschichte 301.

99 Labib, Handelsgeschichte 319 et 421.

100 Frescobaldi, Visit 43. Pour les monuments historiques qu' on pouvait encore y voir en 1908, voir Massignon, Note 20-22 (la plus ancienne inscription relevée par Massignon fut trouvée sur le minbar de la mosquée al-Sabāc. Elle datait de 817/1414-1415).

101 Frescobaldi, Visit 165. Brancacci, en 1422, mentionne lui aussi un arrêt à Fuwwa, pour se rafraîchir et se reposer, jusqu'au départ pour Le Caire au moment des vêpres. Voir Brancacci, Diario 169. Les passagers sont censés emporter leurs victuailles pour toute la durée du voyage. Voir Khalilieh, Islamic 56; Khalilieh, Admiraly 75. Ibn Baț̣ūța apporte des précisions à ce sujet: «Celui qui navigue sur le Nil n'a pas besoin d'emporter des provisions de route, car toutes les fois qu' il veut descendre sur le bord du fleuve, il peut le faire, soit pour vaquer à ses ablutions ou à la prière, soit pour acheter des vivres et autres objets. Des marchés se suivent sans interruption depuis la ville d'Alexandrie jusqu' au Caire, et depuis le Caire jusqu'à la ville d'Assouan». Apud Wiet, Les communications 246. Dans le cas de voyageurs étrangers au dār al-islām, on comprend qu'il était plus intéressant pour eux de partir avec leurs provisions de bouche acquises à Alexandrie, car ils étaient conscients que le prix des marchandises serait immanquablement plus élevé en chemin eu égard à leur origine. 
font état d'un transport fluvial dans une même embarcation d'Alexandrie au Caire pour la simple raison que la saison y était propice.

S' agissant de la durée d' un tel périple, les différentes sources font état d' une moyenne située entre 5 à 6 jours ${ }^{102}$, mais il n'était pas rare d'arriver à destination au bout de 8 jours. L'état de navigabilité du canal, la saison, le climat, la direction et d' autres facteurs externes pouvaient raccourcir ou allonger cette durée. En 1384, Frescobaldi et ses compagnons quittèrent Alexandrie le 5 octobre et arrivèrent au Caire dans la nuit ( $2 \mathrm{~h}$ du matin) du 11 du même mois. Toutefois, dès le 6 octobre au soir, donc le lendemain de leur départ, ils arrivaient à Fuwwa, et ce en comptant le trajet effectué à dos de mule pour rejoindre le canal ${ }^{103}$. En 1422, Brancacci ne mit pas plus de temps pour parcourir la même distance. Il arriva au Caire après seulement quatre jours de voyage ${ }^{104}$. Par contre, en 1447, un pèlerin musulman, al-Qalașādī, mit 8 jours pour rallier Būlāq au départ d'Alexandrie ${ }^{105}$.

Quant au coût d'un tel voyage, les sources donnent aussi de précieuses informations, même si elles doivent être recoupées avec d' autres données. Dans les documents de la Genizah, les frais mentionnés font toujours état d'une somme globale dont il est difficile de retrancher avec précision le coût du transport. Le frêt des marchandises comprenait, en effet, la plupart du temps, le prix $\mathrm{du}$ transport de la personne accompagnante ${ }^{106}$. Notre document ne s'écarte pas de cette pratique: le soldat paie 5 ducats pour le transport de sa propre personne, ses effets personnels et une quantité de textile. En guise de comparaison, Frescobaldi et ses 11 compagnons de voyage payèrent, en 1384, pour le trajet Alexandrie-Le Caire un total de 8 ducats, soit $3 / 4$ de ducat par personne. Deux ans avant notre soldat, Brancacci donna, pour deux personnes, 1 ducat et 8 soldi pour le voyage jusqu'à Fuwwa ${ }^{107}$ et 6 ducats, 2 livres et 8 soldi pour le trajet Fuwwa-Le Caire ${ }^{108}$. En 1481, Meshullam de Volterre, juif italien, paya 30 mu'ayyadī-s ${ }^{109}$ pour le voyage en bateau de Fuwwa au Caire. Seize ans plus tard, Arnold von Harff acquitta un ducat pour le voyage par bateau de Rosette

\footnotetext{
102 Wiet, Les Communications 244; Goitein, A Mediterranean society 1:299.

103 Goitein, A Mediterranean society 1:43-45, 96-98.

104 Brancacci, Diario 169 (départ d'Alexandrie le 30 juillet, arrivée à Fuwwa le 31).

105 Qalașādī, Riḥla 125-126 (départ le 8 jum. II 851/21 août 1447, arrivée le 16 jum. II/29 août 1447).

106 Goitein, A Mediterranean society 1:341.

107 Brancacci, Diario 329.

108 Brancacci, Diario 329.

109 En 1479, 25 mu'ayyadī-s équivalaient à 1 dīnār ashrafí. Ashtor, The Kārimī 279.
} 
au Caire ${ }^{110}$. Ces montants, qui s'étalent sur plus d'un siècle, permettent de constater que le coût moyen du transport de Fuwwa au Caire oscillait entre un à trois ducats, la différence devant s' expliquer par les marchandises qui accompagnaient les voyageurs. On constate donc, pour notre document, que le soldat paya un prix largement supérieur à cette moyenne, ce qui laisse sous-entendre que le textile qu'il transportait devait représenter une part importante dans le calcul du coût du transport. Les données que fournissent les documents de la Genizah nous permettent d'imaginer que la quantité de textile transportée devait être conséquente. Ainsi, pour l' envoi d'une bale d'indigo d'une valeur de 66 dīnārs et 1/4 du Caire à Rosette au XI ${ }^{\mathrm{e}}$ siècle, l' expéditeur dû débourser 3 dìnārs $( \pm 5 \%)$ pour les frais liés à l' expédition et 1 dìnār $(=1,5 \%)$ pour le transport ${ }^{111}$. Ces pourcentages ne peuvent évidemment pas être invoqués pour du textile transporté en 1420, mais ils nous autorisent au moins à considérer que le textile emporté par le soldat représentait une somme importante.

Reste à savoir pourquoi un militaire en poste à Alexandrie qui se rendait au Caire transportait une telle quantité de textile et comment ce contrat a pu finir parmi les documents soit du consul de Venise de l'époque, Biagio Dolfin, soit de son neveu, Lorenzo Dolfin, qui faisait office de vice-consul. Les deux questions sont, à mon humble avis, liées. Biagio Dolfin avait été nommé consul de la République de Saint-Marc à Alexandrie en 1418. Son mandat de deux ans, comme à l' accoutumée ${ }^{112}$, devait donc prendre fin en 1420. En avril de cette même année, nous savons qu'il se trouvait au Caire pour affaire. C'est dans le courant de ce mois qu'il a dû y contracter la peste, maladie à laquelle il ne survécut pas: il trépassa à la fin du mois, son testament étant daté du 27 avril $1420^{113}$. Il n' est pas impossible que son départ survint au tout début d'avril, à la même date que celle de notre document. Il faisait d'ailleurs peut-être partie des passagers qui se trouvaient sur la barque qui devait emmener le soldat de Fuwwa à la capitale ${ }^{114}$. Si le contrat a fini entre ses papiers ou ceux de son neveu, cela signifie que le soldat rendait un service

\footnotetext{
110 Ashtor, The Kārimī 366.

111 Goitein, A Mediterranean society 1:343.

112 Ashtor, The Kārimī 413.

113 Le testament fut rédigé le lendemain de sa mort. Pedani, The Mamluk 140-141. Voir aussi désormais Christ, Trading conflict 101.

114 Dans ce cas, la lecture wa-l-anfär proposée pour la l. 5 se justifierait. Le prix de cinq ducats pourrait alors correspondre au tarif exigé pour le transport de toutes ces personnes et de leurs effets. En 1561, Alessandro Magno paya cinq ducats pour louer une germe qui devait le transporter avec ses dix compagnons de Rosette à Būlāq (Magno, Voyages 263).
} 
à l'un des deux ${ }^{115}$. Ce service consistait probablement soit à les escorter, soit à prendre en charge une quantité de textile qui devait être revendue au Caire, soit encore les deux ${ }^{116}$. Le fait de confier de la marchandise à un passager musulman pourrait sembler étrange et pourtant un document de la Genizah démontre que c'était une pratique sinon courante, du moins attestée. Dans celui-ci, un marchand indien conseille à son frère de confier ses biens à un voyageur musulman afin d'éviter de payer des taxes douanières additionnelles que l'on appliquait aux non-musulmans ${ }^{117}$. Ce stratagème était de bonne guerre, si l'on ose dire: le non-musulman sortait gagnant de cet échange momentané tout autant que le musulman qui se prêtait au jeu, car on imagine qu'il en tirait un avantage pécunier. De ce genre de contrat, nous ne risquons pas de trouver trace.

115 Lorenzo Dolfin, après la mort de son oncle, poursuivit sa carrière de marchand comme l'attestent ses comptes. Voir Ashtor, The Kārimī 431.

116 Dans la première moitié $\mathrm{du} \mathrm{xv}^{\mathrm{e}}$ siècle, l'industrie textile d'Alexandrie était déjà sur le déclin. On en trouve la preuve dans l'augmentation des importations de textiles des Flandres, d'Italie, du Languedoc et d'ailleurs dont étaient responsables les Vénitiens. Voir Ashtor, The Kārimī 270. Le témoignage d'un contemporain comme l'était Piloti est éclairant à plus d'un titre. Parlant des marchandises importées à Alexandrie, au Caire, à Beyrouth et à Damas, il déclare: «Et premièrement draps de laine de Flandres, de Cathalogne, de Barseloigne, et de Venise». Voir Piloti, Traité 107. Pour les exportations de tissus de Catalogne, voir Coulon, Barcelone 312-338.

117 Goitein, A Mediterranean society 1:344-345. Les harbì-s devaient payer un droit de passage à la frontière. Frescobaldi et ses compagnons, en 1384, payèrent chacun un droit de passage de 4 ducats à la sortie d'Alexandrie et un autre d'un ducat par tête à l' approche du Caire. Gucci signale que ces droits de passage rapportaient au sultan quelque cinquante mille florins chaque année. Frescobaldi, Visit 42, 98-99, 165. Il est possible que le droit payé à l' approche du Caire ait en fait correspondu à la taxe de circulation sur le Nil (himāya) qui était due par chaque passager. Elle fut annulée sous le règne du sultan al-Nāșir Muhammad ibn Qalāwūn (r. 1309-1341), puis réintroduite à sa mort. Labib, Handelsgeschichte 251. Al-Maqrīzī fait aussi état d'une taxe non islamique (maks) payable au port de Būlāq et annulée par le même sultan. Voir Maqrīīi al-Mawā'iz wa-l-ítibār 1:88-89. En outre, les marchands non musulmans se voyaient souvent imposer des frais de douane doubles. Goitein (A Mediterranean society 1:344-345) estime que cela ne semble pas avoir été d' application sous les Fatimides, mais que ce fut bien le cas pendant une brève période sous le règne de Saladin. 


\section{Bibliographie}

\section{Sources primaires}

al-Asyūṭī (d. 849/1445), Jawāhir al-'uqūd wa-muìn al-quḍāt wa-l-muwaqqi'ìn wa-lshuhūd, 2 vols. Édité par Mus'ad 'Abd al-Hamīd Muhammad al-Sa'danī, Beyrouth 1417/1996.

Brancacci, F. (d. ca. 1440), Diario di Felice Brancacci, Ambasciatore con Carlo Federighi al Cairo per il Comune di Firenze (1422), Édité par D. Catellaci, Archivio storico italiano VIII, quarta serie (1881): 157-188, 326-334.

Frescobaldi, L., G. Gucci et S. Sigoli, Visit to the holy places of Egypt, Sinai, Palestine and Syria in 1384, Traduit de l'italien par F. Theophilus Bellorini, O.F. Eugene Hoade, \& F. Eugene Hoade, Jérusalem 1948.

Ibn Hijja al-Hamawī (d. 837/1434), Das Rauschgetränk der Stilkunst oder Qahwat alinshā' von Taqūyuddīn Abū Bakr ibn 'Al̄̄ ibn Hiğğa al-Hamaw̄̄ al-Azrārī, Édité par Rudolf Veselý, Berlin 1426/2005.

Magno, A., Voyages (1557-1565), Traduction et notes de W. Naar, Paris 2002.

al-Makhzūmī (d. 585/1189), Al-Minhājfì ilm kharāj Miṣr, Édité par Cl. Cahen et Y. Rāgìib, Le Caire 1986.

al-Maqrīzī (d. 846/1442), Al-Mawāì wz wa-l-ítibār fì dhikr al-khițaṭ wa-l-äthār, 2 vols., Būlāq 1270/1853.

al-Nuwayrī (d. 733/1333), Nihāyat al-arab fí funūn al-adab, 33 vols., Le Caire 1923-1997. Piloti, E. (d. après 1441), Traité d'Emmanuel Piloti sur le passage en Terre Sainte (1420), Édité par Pierre-Herman Dopp, Louvain et Paris 1958.

al-Qalaṣādī (d. 891/1486), Riḥlat al-Qalaṣādī, Édité par Muhammad Abū l-Ajfān, Tunis 1985.

al-Qalqashandī (d. 821/1418), Șubh al-a shhāfís șinā'atal-inshä̀) 14 vols., Le Caire 1913-1920. al-Samarqandī (d. 69o/1291), Kitāb al-Shurūț wa-ulūm al-șukūk, Édité par Muhammad Jāsim al-Hadīthī, Bagdad 1987.

Stochove, V., G. Fermanel et R. Fauvel, Voyage en Égypte, Édité par Baudouin van de Walle, Le Caire 1975 .

\section{Sources secondaires}

Amari, M., I diplomi Arabi del R. archivio Fiorentino, Florence 1863.

al-Ashqar, M. 'A. al-Gh., Tujjār al-tawābil fì Mișr fì al-'așr al-mamlūkī, Le Caire 1999.

Ashtor, E., The Kārimī merchants, in Journal of the Royal Asiatic Society (1956), 45-56. Levant trade in the later Middle Ages, Princeton 1983.

Bacharach, J.L., The dinar versus the ducat, in International Journal of Middle East Studies 4 (1973), 77-96.

Bauden, F., The Mamluk documents of the Venetian state archives: Handlist, in Quaderni di Studi Arabi 20-21 (2002-2003), 147-156. 
- L'Achat d' esclaves et la rédemption des captifs à Alexandrie d'après deux documents arabes d'époque mamelouke conservés aux Archives de l'état à Venise (ASVe), in A-M. Eddé et E. Ganagé (éd.), Regards croisés sur le Moyen Âge arabe. Mélanges à la mémoire de Louis Pouzet s.J. (1928-2002), Beyrouth 2005, 269-325.

- The role of interpreters in Alexandria in the light of an oath (Qasāma) taken in the year 822 A.H./1419A.D., in A. van Tongerloo et K. D'hulster (éd.), Tradition and change in the realms of Islam. Studies in honour of professor Urbain Vermeulen, Louvain 2008, 33-63.

Christ, G., Trading conflict. Venetian merchants and Mamluk officials in late medieval Alexandria, Leyde et Boston 2012.

Colin, G.S., Notes de dialectologie arabe. II. Technologie de la batellerie du Nil, in Bulletin de l'Institut français d'archéologie orientale 20 (1922), 45-87.

Combe, É., Alexandrie musulmane. Notes de topographie et d'histoire de la ville depuis la conquête arabe jusqu'à nos jours, in Bulletin de la Société de Géographie d'Égypte XVI (1929), 111-171, 269-292.

Coulon, D., Barcelone et le grand commerce d'Orient au Moyen Âge. Un siècle de relations avec l'Égypte et la Syrie-Palestine (ca. 1330 - ca. 1430), Madrid et Barcelone 2004.

Diem, W. et H.P. Radenberg, A dictionary of the Arabic material of S.D. Goitein's A Mediterranean society, Wiesbaden 1994.

Dozy, R.P., Supplément aux dictionnaires arabes, 2 vols., Leyde 1881, réimpr. Beyrouth 1981.

Fischel, W.J., Über die Gruppe der Kārimī-Kaufleute. Ein Beitrag zur Geschichte des Orienthandels Ägyptens unter den Mamluken, in Studia Arabica (Analecta Orientalia) 1 (1937), 65-82.

- The spice trade in Mamluk Egypt. A contribution to the economic history of medieval Islam, in Journal of the Economic and Social History of the Orient 1 (1958), 157-174.

Goitein, S.D., New light on the beginnings of the Kārim merchants, in Journal of the Economic and Social History of the Orient 1 (1958), 175-184.

A Mediterranean society. The Jewish communities of the Arab world as portrayed in the documents of the Cairo geniza, 6 vols., Berkeley 1967 .

Graboïs, A., La Description de l'Égypte au XIv e siècle par les pèlerins et les voyageurs occidentaux, in Le Moyen Âge (Revue d'histoire et de philologie) CIX (2003), 529-543.

Guest, A.R., The delta in the Middle Ages. A note on the branches of the Nile and the kurahs of lower Egypt, with map, in Journal of the Royal Asiatic Society (1912), 941-980.

Guo, L., Commerce, culture, and community in a Red Sea port in the thirteenth century. The Arabic documents from Qusayr, Leyde et Boston 2004.

Hairy, I. et O. Sennoune, Géographie historique du canal d'Alexandrie, in Annales Islamologiques 40 (2006), 247-278. 
Halflants, B., Le Conte du Portefaix et des Trois Jeunes Femmes dans le manuscrit de Galland (XIV $-X V^{e}$ siècles): Édition, traduction et étude du moyen arabe d'un conte des Mille et une nuits, Louvain-la-Neuve 2007.

Hopkins, S., Studies in the grammar of early Arabic: Based upon papyri datable to before 30oA.H./912 A.D., Oxford 1984.

Kahle, P., Zur Geschichte des mittelalterlichen Alexandria, in Der Islam 12 (1922), 29-83.

Khalilieh, H.S., Islamic maritime law. An introduction, Leyde, Boston et Cologne 1998. Admiralty and maritime laws in the Mediterranean Sea (ca. 800-1050): The Kitāb Akriyat Al-Sufun vis-à-vis the Nomos Rhodion Nautikos, Leyde et Boston 2006.

Khoury, R.G., Chrestomathie de papyrologie arabe. Documents relatifs à la vie privée, sociale et administrative dans les premiers siècles islamiques, Leyde, New York et Cologne 1993.

Kindermann, H., «Schiff» im Arabischen. Untersuchung über Vorkommen und Bedeutung der Termini, Ph.D., Friedrich-Wilhelms-Universität zu Bonn 1934.

Labīb, Ṣ., Al-Tijāra al-kārimīya wa tijārat Miṣr fī l-'uṣūr al-wusțā, in al-Majalla al-Tảrīkhìya al-Mișrīya 4 (1952), 5-63.

Labib, S.Y., Handelsgeschichte Ägyptens im Spätmittelalter (1171-1517), Wiesbaden 1965. Les Marchands kārimīs en Orient et sur l'océan indien, in M. Mollat (éd.), Sociétés et compagnies de commerce en Orient et dans l'océan indien. Actes du huitième colloque international d'histoire maritime (Beyrouth - 5-10 Septembre 1966), Paris 1970, 209-214.

Lane, E.W., Madd al-Qāmūs. An Arabic-English lexicon, 8 vols., Londres et Edimbourg 1863, réimpr. Beyrouth 1968.

Little, D.P., A catalogue of the Islamic documents from al-Haram al-Sharif in Jerusalem, Beyrouth 1984.

Malti-Douglas, F., The interrelationship of onomastic elements: Isms, Dîn-Names and Kunyas in the ninth century A.H., in Cahiers d'onomastique arabe 1981 (1982), 27-55. Maspero, J. et G. Wiet, Matériaux pour servir à la géographie de l'Égypte. Première série: Liste des provinces, villes et villages d'Égypte citées dans les tomes I et II des Khițtat de Maqrîzî, Le Caire 1919.

Massignon, L., Note sur l'état d'avancement des études archéologiques arabes en Égypte hors du Caire, in Bulletin de l'Institut français d'archéologie orientale 6 (1908), $1-24$.

al-Nakhīlī, D., Al-Sufun al-islāmìya 'alā ḥurūfal-mu'jam, s.l. [Le Caire] 1974.

Pedani, M.P., The Mamluk documents of the Venetian state archives: Historical survey, in Quaderni di Studi Arabi 20-21 (2002-2003), 133-146.

Popper, W., Egypt and Syria under the Circassian sultans, 1382-1468A.D. Systematic notes to Ibn Taghrî Birdîs chronicles of Egypt, 2 vols., Berkeley et Los Angeles 1955-1957.

Rāgib, Y., Actes de vente d'esclaves et d'animaux d'Égypte médiévale, Le Caire 2006. 
Rayner, S.E., The theory of contracts in Islamic law: A comparative analysis with particular reference to the modern legislation in Kuwait, Bahrain and the United Arab Emirates, Londres, Dordrecht et Boston 1991.

Sijpesteijn, P.M., Travel and trade on the river, in P.M. Sijpesteijn et L. Sundelin (éd.), Papyrology and the history of early Islamic Egypt (Islamic history and civilization. Studies and texts vol. 55), Leyde et Boston 2004, 115-152.

Sublet, J., 'Abd al-Lațîf al-Takrītī et la famille des Banū Kuwayk, marchands kārimī, in Arabica 9 (1962), 193-196.

Toussoun, O., Mémoire sur les anciennes branches du Nil. Époque ancienne et arabe, Le Caire 1922.

. Mémoire sur l'histoire du Nil, 3 vols., Le Caire 1925.

Udovitch, A.L., Time, the sea and society: Duration of commercial voyages on the southern shores of the Mediterranean during the high Middle Ages, in La Navigazione mediterranea nell'alto medioevo, Spoleto 1978, 503-545.

Von Kremer, A.F., Beiträge zur arabischen Lexikographie, 2 vols., Vienne 1883.

Wehr, H., A dictionary of modern written Arabic, Édité par J. Milton Cowan, Ithaca 1976.

Wiet, G., Les Communications en Égypte au Moyen Âge, in L'Égypte contemporaine 24 (1933), 241-264.

. Les Marchands d'épices sous les sultans mamlouks, in Cahiers d'histoire égyptienne 7 (1955), 81-147.

Zanetti, U., Filigranes vénitiens en Égypte, in L.S. Olschki (ed.), Studi albanologici, balcanici, bizantini e orientali in onore di Giuseppe Valentini, s.J., Florence 1986, 437-499. 